\title{
Latest results on the analysis of the radio frequency spectrum emitted by high energy air showers with LOFAR
}

Laura Rossetto $^{1, \star}$, Antonio Bonardi ${ }^{1}$, Stijn Buitink ${ }^{2}$, Arthur Corstanje ${ }^{1}$, Heino Falcke ${ }^{1,3,4}$, Brian M. Hare ${ }^{5}$, Jörg R. Hörandel ${ }^{1,3}$, Pragati Mitra ${ }^{2}$, Katie Mulrey², Anna Nelles ${ }^{6,7}$, Jörg P. Rachen ${ }^{1}$, Pim Schellart ${ }^{8}$, Olaf Scholten ${ }^{5,9}$, Satyendra Thoudam ${ }^{10}$, Gia T.N. Trinh ${ }^{5}$, Sander ter Veen ${ }^{4}$, and Tobias Winchen ${ }^{2}$

${ }^{1}$ Department of Astrophysics/IMAPP, Radboud University, P.O. Box 9010, 6500 GL Nijmegen, The Netherlands

${ }^{2}$ Astrophysical Institute, Vrije Universiteit Brussel, Pleinlaan 2, 1050 Brussels, Belgium

${ }^{3}$ NIKHEF, Science Park Amsterdam, 1098 XG Amsterdam, The Netherlands

${ }^{4}$ Netherlands Institute of Radio Astronomy (ASTRON), Postbus 2, 7990 AA Dwingeloo, The Netherlands

${ }^{5} \mathrm{KVI}-\mathrm{CART}$, University Groningen, P.O. Box 72, 9700 AB Groningen, The Netherlands

${ }^{6}$ DESY, Platanenallee 6, 15738 Zeuthen, Germany

${ }^{7}$ Humboldt-Universität zu Berlin, Institut für Physik, Newtonstrasse 15, 12489 Berlin, Germany

${ }^{8}$ Department of Astrophysical Sciences, Princeton University, Princeton, NJ 08544, USA

${ }^{9}$ Interuniversity Institute for High-Energy, Vrije Universiteit Brussel, Pleinlaan 2, 1050 Brussels, Belgium

${ }^{10}$ Department of Physics and Electrical Engineering, Linnéuniversitetet, 35195 Växjö, Sweden

\begin{abstract}
The LOw Frequency ARay (LOFAR) is a multi-purpose radio antenna array aimed to detect radio signals in the frequency range $10-240 \mathrm{MHz}$, covering a large surface in Northern Europe with a higher density in the Netherlands. Analytical calculations and simulation studies performed in the 2000s indicate a dependence of the radio frequency spectrum on cosmic-ray characteristics. The high number density of radio antennas at the LOFAR core allows to characterise the observed cascade in a detailed way. The radio signal emitted by air showers in the atmosphere has been studied accurately in the $30-80 \mathrm{MHz}$ frequency range. The analysis has been conducted on simulated events and on real data detected by LOFAR since 2011. The final aim of this study is to find an independent method to infer information of primary cosmic rays for improving the reconstruction of primary particle parameters. Results show a strong dependence of the frequency spectrum on the distance to the shower axis for both real data and simulations. Furthermore, results show that this method is very sensitive to the precision in reconstructing the position of the shower axis at ground, and to different antenna calibration procedures. A correlation between the frequency spectrum and geometrical distance to the shower maximum development $X_{\max }$ has also been investigated.
\end{abstract}

\section{Analysis and Results}

In order to study a correlation between the radio frequency spectrum and cosmic-ray air shower characteristics, the radio signal in the frequency-domain has been investigated by analysing data detected

^e-mail: 1.rossetto@astro.ru.nl 
by LOFAR [1] since 2011, and simulated events generated with CORSIKA (version 7.6300) [2] and CoREAS [3]. The following method has been applied to both real data and simulations:

- the antenna signal in the time-domain is converted into the frequency-domain by applying a Fast Fourier Transform (FFT). The FFT is calculated on a time window of $640 \mathrm{~ns}$ defined as $\left[\mathrm{t}_{0}-240 \mathrm{~ns}\right.$, $\left.\mathrm{t}_{0}+400 \mathrm{~ns}\right]$ around the signal peak time $\mathrm{t}_{0}$; the $|\mathrm{FFT}|^{2}$ signal is then evaluated;

- the background is calculated on a longer time window of $328 \mu$ s, centred on the pulse peak; this time window is divided in about 400 sub-windows of $640 \mathrm{~ns}$ each, after having excluded the pulse region. The $|\mathrm{FFT}|^{2}$ background is calculated by averaging the $|\mathrm{FFT}|^{2}$ of each of these sub-windows;

- the final $|\mathrm{FFT}|^{2}$ is evaluated as $|\mathrm{FFT}|^{2}=|\mathrm{FFT}|^{2}$ signal $-|\mathrm{FFT}|^{2}$ background

- a linear fit is then applied to $\log _{10}|\mathrm{FFT}|^{2}$ in the frequency range $33-70 \mathrm{MHz}$.

Since the analysis is very sensitive to different parameters, strict selection criteria have been applied in order to select a clean event sample:

- at least 1 station with half of available antennas having signal $>10 \sigma$

- events with at least 10 antennas having $\left|\mathrm{FFT}\left(v_{i}\right)\right|^{2}>\operatorname{RMS}\left(\left|\mathrm{FFT}\left(v_{i}\right)\right|^{2}\right.$ background $)$

Results show that the linear-fit slope of the frequency spectrum strongly depends on the distance to the shower axis for both real data and simulations, as depicted in Fig. (1). In particular, the spectrum follows a parabolic function with a maximum around $100 \mathrm{~m}$ in agreement with the Cherenkov ring region. Furthermore, it has been found that the linear-fit slope as function of distance to the shower axis is extremely sensitive to the selected frequency range. Analysis performed on simulated events shows that the slope measured in the $30-70 \mathrm{MHz}$ range is on average approximately $0.003 \pm 0.001 \mathrm{MHz}^{-1}$ steeper than the slope measured in the $30-75 \mathrm{MHz}$ range.

The frequency spectrum depends also on the geometrical distance of the observer to $\mathrm{X}_{\max }$ (hereafter, $D_{\max }$ ), as shown for simulations in Fig. (2). Moreover, as depicted in Fig. (3) at a given distance to the shower axis the linear-fit slope is correlated to $\mathrm{D}_{\max }$ by the following function: $\mathrm{p}_{0} \cdot \exp \left(-\mathrm{p}_{1} \cdot \mathrm{D}_{\max }\right)+\mathrm{p}_{2}+\mathrm{p}_{3} \cdot \mathrm{D}_{\max }$. Final results on the dependence of the linear-fit slope as function of $\mathrm{D}_{\max }$ for real data are still under investigation, and they will be discussed in a forthcoming publication.

\section{Conclusions}

The radio frequency spectrum has been studied in the range $30-80 \mathrm{MHz}$ (for more details see [4]). A clear dependence of the frequency spectrum as function of distance to the shower axis has been obtained. Moreover, at fixed distances from the shower axis, the frequency spectrum depends also on the geometrical distance to $\mathrm{X}_{\max }$. It is important to underline that values of the linear-fit slope are biased by changing the frequency range and the antenna calibration method. Anyhow, the general dependence of the slope as function of distance to the shower axis and $\mathrm{X}_{\max }$ is not affected.

\section{References}

[1] M.P. van Haarlem et al., A\&A 556, A2, 53 (2013)

[2] D. Heck et al., Report FZKA 6019 (1998)

[3] T. Huege, M. Ludwig, and C.W. James, AIP Conference Proceedings 1535, 128-132 (2013)

[4] L. Rossetto, in proceedings of $35^{\text {th }}$ ICRC 2017 Conference PoS(ICRC2017)329 (2017) 

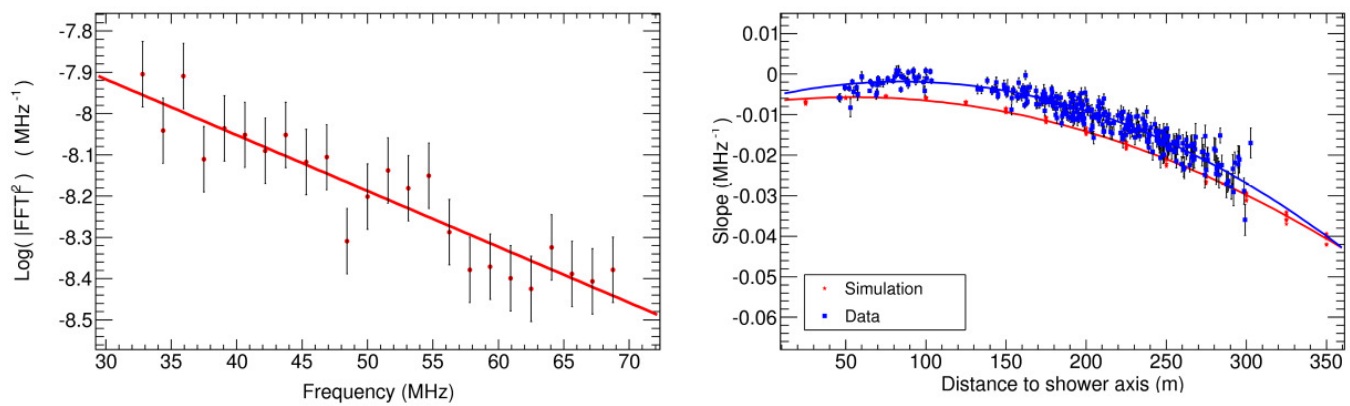

Figure 1. Left: distribution of $\log _{0} \mid \mathrm{FFT} P$ as function of frequency for one antenna of one selected event; the distribution has been fitted with a linear function, taking into account $20 \%$ uncertainty on the $|\mathrm{FFT}|^{2}$ converted into logarithmic values. Right: distribution of the linear-fit slope as function of distance to the shower core for one selected event (blue squares) and the corresponding simulated event (red stars). Both distributions have been fitted with a parabolic function.
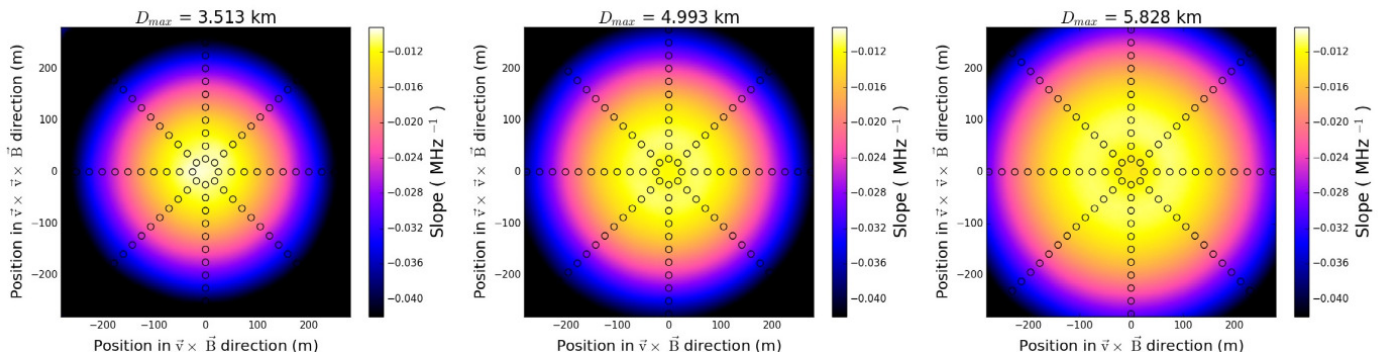

Figure 2. Distribution of the linear-fit slope on the shower plane for three simulated events with different values of $\mathrm{D}_{\max }$. Slope values of the 160 antennas (open circles) used for the simulation procedure have been interpolated in order to create a smooth background.
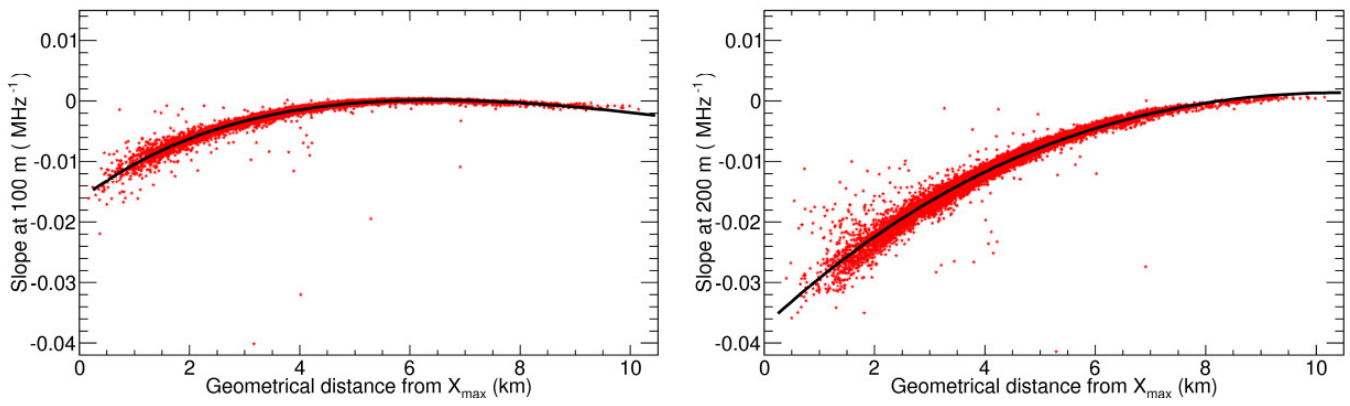

Figure 3. Distribution of the linear-fit slope (red stars) for simulated events as function of $\mathrm{D}_{\max }$ at $100 \mathrm{~m}$ (left) and $200 \mathrm{~m}$ (right) from the shower axis. Both distributions have been fitted with a 4-parameters function: $\mathrm{p}_{0} \cdot \exp \left(-\mathrm{p}_{1} \cdot \mathrm{D}_{\max }\right)+\mathrm{p}_{2}+\mathrm{p}_{3} \cdot \mathrm{D}_{\max }$. 